\title{
A Hybrid Econometric-AI Ensemble Learning Model for Chinese Foreign Trade Prediction
}

\author{
Lean $\mathrm{Yu}^{1,2}$, Shouyang Wang ${ }^{1}$, and Kin Keung $\mathrm{Lai}^{2}$ \\ ${ }^{1}$ Institute of Systems Science, Academy of Mathematics and Systems Science, \\ Chinese Academy of Sciences, Beijing 100080, China \\ \{yulean, sywang\} @amss.ac.cn \\ ${ }^{2}$ Department of Management Sciences, City University of Hong Kong, \\ Tat Chee Avenue, Kowloon, Hong Kong \\ \{mskklai, msyulean\} @cityu. edu.hk
}

\begin{abstract}
Due to the complexity of economic system, the interactive effects of economic variables or factors on Chinese foreign trade make the prediction of China's foreign trade extremely difficult. To analyze the relationship between economic variables and foreign trade, this study proposes a novel nonlinear ensemble learning methodology hybridizing nonlinear econometric model and artificial neural networks (ANN) for Chinese foreign trade prediction. In this proposed learning approach, an important econometrical model, the cointegration-based error correction vector auto-regression (EC-VAR) model is first used to capture the impacts of the economic variables on Chinese foreign trade from a multivariate analysis perspective. Then an ANN-based EC-VAR model is used to capture the nonlinear patterns hidden between foreign trade and economic factors. Subsequently, for introducing the effects of irregular events on foreign trade, the text mining and expert's judgmental adjustments are also incorporated into the nonlinear ANN-based EC-VAR model. Finally, all economic variables, the outputs of linear and nonlinear EC-VAR models and judgmental adjustment model are used as another neural network inputs for ensemble prediction purpose. For illustration, the proposed ensemble learning methodology integrating econometric techniques and artificial intelligence (AI) methods is applied to Chinese export trade prediction problem.
\end{abstract}

Keywords: Artificial neural networks, error-correction vector auto-regression, hybrid ensemble learning, foreign trade prediction.

\section{Introduction}

Since the reforms and opening policies was initiated in 1978, China has attracted foreign investment, brought in foreign advanced technology, increased commodity exports and accelerated economic growth. Currently, China is the world's fastest developing nation. If it grows at an average of $4 \%$ to $6 \%$ annually, China will overtake Japan as Asia's largest economy by 2040 [1]. In China's economy, the most dynamic and important part is the external sector. In 2003, the openness of economy (the ratio of foreign trade in GDP) exceeds 60 percent. About one-fourth of China's 
industrial labors are employed by the foreign trade related industries [2]. Thus China's government pays a great attention to foreign trade.

As a transition economy, China's government has been playing a key role in its economic development. For accelerating the development of foreign trade, the government not only has issued a series of favorable policies. One important policy is "export rebate tax". Every March of the year, the Chinese highest legislation authority, the National People's Congress (NPC), will hold its annual grand session. In this session, the delegates will audit the fiscal plan of the central government. The "export rebate tax" was an important part in the fiscal plan. To make a feasible fiscal plan, it is necessary to make an accurate prediction for foreign trade. Therefore, foreign trade forecasting has an important implication for Chinese macroeconomic policymaking. For example, if export trade forecasting is accurate, the national fiscal program about "export rebate tax" is easy to be made and to be implemented. If forecasting is larger than the actual exports, some financial fund will be otiose and cannot exert its function. If forecasting is smaller than the actual exports, the financial subsidies will not be sent to export enterprises and thus affecting their enthusiasm. So, accurate foreign trade forecasting can reduce uncertainty and blindness in macroeconomic policymaking and obtain good economic benefit

However, it is difficult to predict the foreign trade accurately due to the complexity of economic system and interactive effects of the economic variables. For this purpose, this paper attempts to propose a nonlinear ensemble learning methodology hybridizing nonlinear econometric model and artificial neural networks (ANN) for Chinese foreign trade prediction. In this proposed learning approach, an important econometrical model, the co-integration-based error correction vector auto-regression (EC-VAR) model is first used to capture the impacts of the economic variables on Chinese foreign trade from a multivariate analysis perspective. Then an ANN-based EC-VAR model is used to capture the nonlinear patterns hidden between foreign trade and economic factors. Subsequently, for introducing the effects of irregular events on foreign trade, the text mining and expert's judgmental adjustments are also incorporated into the nonlinear ANN-based EC-VAR model. Finally, all economic variables, the outputs of linear and nonlinear EC-VAR models and judgmental adjustment model are used as another neural network inputs for ensemble purpose.

The major aim of this study is to present a new hybrid econometric-AI ensemble learning methodology that can significantly improve the prediction performance and thus improving their effectiveness on complex economic prediction problems. The rest of this study is organized as follows. Section 2 describes the building process of the proposed hybrid econometric-AI ensemble learning method in detail. For further illustration, the proposed ensemble learning methodology is applied to Chinese export trade prediction in Section 3. Finally, some conclusions are drawn in Section 4.

\section{The Hybrid Econometric-AI Ensemble Learning Methodology}

In this section, an overall formulation process of the hybrid econometric-AI ensemble learning methodology is described. First of all, the co-integration technique and error correction vector auto-regression (EC-VAR) model are briefly reviewed. Then an ANN-based EC-VAR model is presented to explore nonlinear patterns in the complex 
economic phenomena. To incorporate the effects of irregular events into prediction, text mining and expert's judgmental adjustments are also considered into the ANNbased EC-VAR model. In order to integrate almost all information and implied knowledge, another neural network model is used to realize the nonlinear ensemble.

\subsection{Co-integration Technique and EC-VAR Model}

At one time conventional wisdom was that non-stationary variables should be differenced to make them stationary before including them in multivariate models. However, this situation was changed when Engle and Granger [3] introduced the concept of cointegration in 1987. They showed that it is quite possible for a linear combination of integrated variables to be stationary. In this case the variables are said to be cointegrated. Consider a set of variables in long-run equilibrium (static equilibrium) when $\beta_{1} x_{1 t}+\beta_{2} x_{2 t}+\ldots+\beta_{n} x_{n t}=0$. The equilibrium error is then $e_{t}=\beta^{T} X_{t}$. If the equilibrium is meaningful it must be the case that the error is stationary. For this, co-integration technique is popular and widely used in many domains since 1987. Interested readers can refer to Engle and Granger [3] for more details.

If co-integration relationship between variables exists, an error correction vector auto-regressive (EC-VAR) model can be formulated for prediction purpose. Actually, an EC-VAR model can be seen as a co-integration-based forecasting model, which is represented by

$$
\Delta y_{t}=\sum_{i=1}^{k_{0}} \alpha_{i} \Delta y_{t-i}+\sum_{j=1}^{m} \sum_{i=0}^{k_{j}} \beta_{j, i} \Delta x_{j, t-i}+\gamma \cdot E C_{t-1}+\xi_{t}
$$

where $y_{t}$ is dependent variable, $y_{t-i}$ is the lag term, $x_{t-i}$ is the lag terms of the independent variables, $E C_{t-1}$ is the co-integration relation, or error correction term, and $\xi_{t}$ is the Gaussian noise, and $\alpha, \beta, \gamma$ are the coefficients of different variables or lag terms, respectively. Generally, an EC-VAR model can lead to a better understanding of the nature of any nonstationarity among the different component series and can also improve longer term forecasting performance over an unconstrained model.

\subsection{ANN-Based Nonlinear EC-VAR Model}

In the above co-integration-based EC-VAR model, the EC is only a linear error correction term. Usually, the errors of component series contain much nonlinearity, a linear error correction is often not sufficient. For this purpose, Hendry and Ericsson [4] proposed a nonlinear EC-VAR model with the following representation:

$$
\Delta y_{t}=\sum_{i=1}^{k_{0}} \alpha_{i} \Delta y_{t-i}+\sum_{j=1}^{m} \sum_{i=0}^{k_{j}} \beta_{j, i} \Delta x_{j, t-i}+\gamma \cdot f\left(E C_{t-1}\right)+\xi_{t}
$$

where $f(\bullet)$ is a nonlinear function, $E C_{t-1}$ denotes a long-term co-integration relationship, and other symbols are similar to Equation (1) . Hendry and Ericsson [4] used a thrice function of $E C_{t-1}$ to predict the currency demand of UK and obtained good performance. In this study, we used ANN to construct a nonlinear function $f(\bullet)$ to create an ANN-based nonlinear EC-VAR model. That is, the proposed ANN-based 
EC-VAR model applies the sigmoid function to determine the nonlinear function $f(\bullet)$ as the error correction term. In this paper, the sigmoid function is represented by

$$
f(E C)=\frac{1}{1+e^{-(w * E C+b)}}
$$

where $w$ and $b$ are unknown parameters, which is determined by ANN training. Usually, the feed-forward neural network (FNN) can realize this goal. Hornik et al. [5] and White [6] found that a three-layer FNN with an identity transfer function in the output unit and logistic functions in the middle-layer units can approximate any continuous function arbitrarily well given a sufficient amount of middle-layer units.

Generally, the ANN-based EC-VAR model is performed in the following steps:

(1) Determine the co-integration relationship between the variables, and

(2) The co-integration relationship is placed into the VAR equation with a nonlinear function form instead of linear function form or a constant. Particularly, this paper uses the sigmoid function as the nonlinear function.

\subsection{Incorporating Text Mining and Judgmental Adjustments into the EC-VAR}

In the complex economic system, the interactive effects are from various sources. Besides the related variables and nonlinear interactions, some irregular events, e.g., 911 terrorist attack, have an important impact on the world economy. To further improve the prediction performance, the text mining and expert's judgmental adjustment are incorporating into the forecasting model. Generally text mining refers to the process of extracting interesting and non-trivial information and knowledge from unstructured text [7]. Interested readers can refer to Sullivan [8] for more details about text mining. In this study, the main goal of the text mining is to retrieve related information from various sources and the human experts provide the judgmental adjustment information for these important information. Within such a framework, the Equation (2) can be extended as

$$
\Delta y_{t}=\sum_{i=1}^{k_{0}} \alpha_{i} \Delta y_{t-i}+\sum_{j=1}^{m} \sum_{i=0}^{k_{j}} \beta_{j, i} \Delta x_{j, t-i}+\gamma \cdot f\left(E C_{t-1}\right)+J A+\xi_{t}
$$

where $J A$ represents the expert's judgmental adjustment for a specified event and other symbols are the same as the above.

\subsection{Nonlinear Ensemble Forecasting Model}

Considering various different economic factors and judgmental adjustments, we have so far formulated three main forecasting equations. In order to capture the effects of different variables and increase forecasting accuracy, it is important to integrate them into a single more accurate forecast. Suppose that there are $k$ lag terms $\left(y_{t-i}, i=1,2, \ldots, k\right)$, $m$ related variables $\left(x_{j}, j=1,2, \ldots, m\right)$, and $p$ forecasts provided by different forecasting equations shown in the previous subsections, then nonlinear ensemble model can be represented as 


$$
\hat{y}_{t}=\varphi\left(y_{t-1}, \cdots, y_{t-k} ; x_{1}, x_{2}, \cdots, x_{m} ; \hat{y}_{1}, \hat{y}_{2} \cdots, \hat{y}_{p} ; w\right)+\xi_{t}
$$

where $w$ is a vector of all parameters and $\varphi(\bullet)$ is a nonlinear ensemble function.

Determining the nonlinear function $\varphi(\bullet)$ is quite challenging. In this study, a standard FNN is employed to realize nonlinear mapping [9]. Actually the paper uses the $k$ lag terms $\left(y_{t-i}, i=1,2, \ldots, k\right), m$ related variables $\left(x_{j}, j=1,2, \ldots, m\right)$, and $p$ forecasts as another neural network inputs to construct the nonlinear ensemble model. Fig. 1 gives an intuitive illustration for the proposed nonlinear ensemble model.

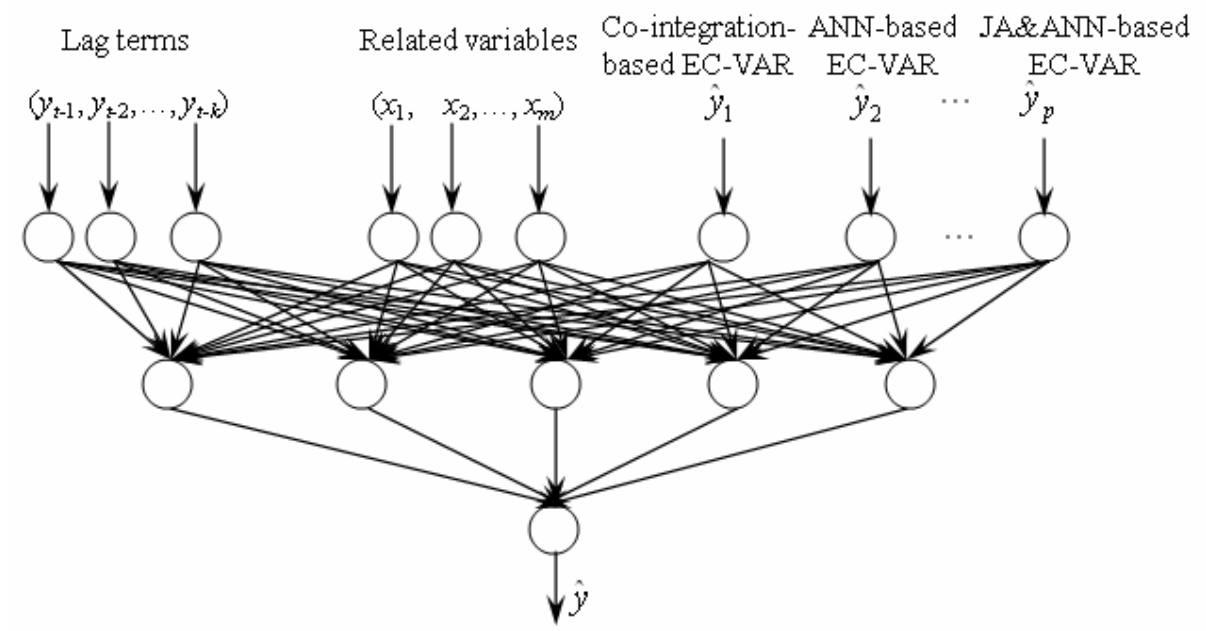

Fig. 1. An intuitive illustration for the nonlinear ensemble forecasting model

It is interesting to examine the underlying idea of the proposed hybrid econometricAI ensemble learning methodology. For a complex and difficult forecasting problem, one single linear or nonlinear model is inadequate to model it due to interactive effect of multiple factors. Through the linear co-integration-based EC-VAR, ANN-based ECVAR and judgmental adjustment-based nonlinear EC-VAR models, the linear patterns, nonlinear patterns, and irregular patterns are also explored. In order to formulate a comprehensive understanding, the explored patterns are aggregated into a single prediction, as indicated in Eq. (5).

From the above analysis, it is obvious that the conventional linear econometric models are insufficient for complex prediction problem because some nonlinear and irregular patterns are not captured by them. From this perspective, it is not hard to understand why conventional linear econometric models cannot always understand and predict some complex and difficult economic problem well. In this sense, using such a hybrid econometric-AI ensemble learning methodology, some complex prediction problems are easy to be implemented based on different linear and nonlinear models as well as nonlinear ensemble technique. 


\section{Experiment}

In order to evaluate the effectiveness of the proposed hybrid econometric-AI ensemble learning methodology, a complex time series prediction task - Chinese foreign trade prediction problem - is investigated. Due to the complexity of economic system, the effects of the economic variables or factors on Chinese foreign trade are realized from many different perspectives. For this, we must select some representative variables to construct a model. The following principle is worth considering in the process of model variable selection. (a) Theoretical interpretability. This requires that the selected variable must have an explanation power for model. That is, the variable can explain why it affects the Chinese foreign trade from the theoretical viewpoint. (b) Representative. This requires us to find some representative variables to construct a Chinese foreign trade forecasting model. That is to say, the selected variables are some important variables affecting Chinese foreign trade. (c) Usability and availability. This principle requires that the related data is available for selected variables. Once choosing the trade partners and competitors, we can select some main variables affecting Chinese foreign trade, as shown in Table 1.

Table 1. Some main factors affecting Chinese foreign trade

\begin{tabular}{|c|c|c|c|}
\hline Category & China & Trade partners & Trade competitors \\
\hline $\begin{array}{l}\text { Export and im- } \\
\text { port indicator }\end{array}$ & $\begin{array}{l}\text { The total amount of } \\
\text { Chinese foreign trade }\end{array}$ & $\begin{array}{l}\text { Import amount of } \\
\text { main trade partners }\end{array}$ & $\begin{array}{l}\text { Export amount of } \\
\text { main trade competi- } \\
\text { tors }\end{array}$ \\
\hline $\begin{array}{l}\text { The nominal } \\
\text { exchange rates } \\
\text { against the US } \\
\text { dollar }\end{array}$ & $\begin{array}{l}\text { Renminbi }(\mathrm{RMB}) \\
\text { exchange rate against } \\
\text { US dollar }\end{array}$ & $\begin{array}{l}\text { Hong Kong dollar, } \\
\text { yen, Germany mark, } \\
\text { Korean won, Singa- } \\
\text { pore dollar, Taiwan } \\
\text { new dollar. }\end{array}$ & $\begin{array}{l}\text { Japanese yen, Korean } \\
\text { won, Taiwan new } \\
\text { dollar, and East Asian } \\
\text { currency rates }\end{array}$ \\
\hline $\begin{array}{l}\text { Economic } \\
\text { situations and } \\
\text { investment } \\
\text { Monetary } \\
\text { policy }\end{array}$ & $\begin{array}{l}\text { Inflation rates, GDP, } \\
\text { foreign direct invest- } \\
\text { ment } \\
\text { Currency supply, for- } \\
\text { eign exchange re- } \\
\text { serve }\end{array}$ & $\begin{array}{l}\text { CPI, customer confi- } \\
\text { dence index, GDP and } \\
\text { unemployment rate }\end{array}$ & $\begin{array}{l}\text { CPI and GDP of these } \\
\text { countries }\end{array}$ \\
\hline
\end{tabular}

In addition, the data used in this study are monthly covered from January 1985 to December 2003, which is from various sources. The data of Chinese export, foreign reserve, and foreign direct investment is collected from Statistical bulletin of the Ministry of Commerce, while the exchange rates data is from Federal Reserve Economic Data of United States (http://www.stls.frb.org/fred/). We take the data from January 1985 to December 1999 with a total of 180 observations as training set and the remainder is used as the testing set (48 observations).

To compare the forecasting performance, the normalized mean squared error (NMSE) is used as the evaluation criterion, which can be represented by

$$
N M S E=\frac{\sum_{i=1}^{N}\left(x_{i}-\hat{x}_{i}\right)^{2}}{\sum_{i=1}^{N}\left(x_{i}-\bar{x}_{i}\right)^{2}}=\frac{1}{\sigma^{2}} \frac{1}{N} \sum_{i=1}^{N}\left(x_{i}-\hat{x}_{i}\right)^{2}
$$


where $\sigma^{2}$ is the estimated variance of the testing data, $x_{i}$ and $\hat{x}_{i}$ are the actual and predicted value, $\bar{x}_{i}$ being the mean, and $N$ is the number of testing data. If the estimated mean of the data is used as predictor, NMSE $=1.0$ is obtained. The NMSE is related to $R^{2}$ which measures the dependence between pairs of desired values and predictions by $N M S E=1-R^{2}$. For comparison, the linear EC-VAR and ANN-based nonlinear EC-VAR model is used as the benchmark model. Particularly, the text mining based judgmental adjustment is also incorporated into ANN-based EC-VAR model. For example, the SARS in April 2003, an important irregular event, has a significant impact on Chinese foreign trade in May and June 2003. Such an event is hard to be included into the model, thus we go to consult the related experts to quantify the effects on Chinese foreign trade. Experts can give a judgmental adjustment value according to their experience. Table 2 reports the empirical results.

Table 2. The out-of-sample prediction results for different prediction models (2000:01-2003:12)

\begin{tabular}{lccc}
\hline \multirow{2}{*}{ Prediction Model } & \multicolumn{3}{c}{ Prediction Performance } \\
\cline { 2 - 4 } & NMSE & Minimum error & $\mathrm{R}^{2}$ \\
\hline Linear EC-VAR & 0.2923 & 0.0102 & 0.7077 \\
ANN-based EC-VAR & 0.1347 & 0.0156 & 0.8653 \\
JA\& ANN-based EC-VAR & 0.0987 & 0.0067 & 0.9013 \\
Hybrid Econometric-AI ensemble & 0.0755 & 0.0000 & 0.9245 \\
\hline
\end{tabular}

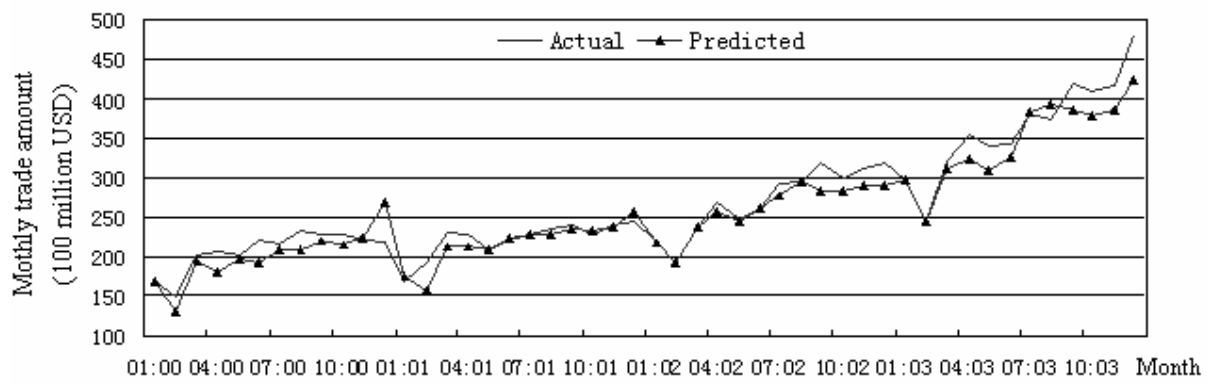

Fig. 2. Prediction results of hybrid econometric-AI ensemble learning model (2000:01-2003:12)

As can be revealed from Table 2, it is not hard to find that the proposed hybrid econometric-AI ensemble learning methodology consistently performs better than the other three prediction models. Furthermore, the minimum error of the proposed hybrid econometric-AI ensemble learning methodology is zero, implying that the proposed methodology is a feasible approach to foreign trade prediction. The main reason leading to good prediction performance may be from the following three aspects. First of all, the hybrid ensemble learning methodology integrates not only linear patterns but also nonlinear patterns as well as the effects of irregular events. Second, the hybrid ensemble learning method makes full use of both the advantage of econometric model, e.g., EC-VAR in this study and the advantages of the AI techniques such as ANN and text 
mining. Third, the final ensemble model applies a nonlinear ensemble learning strategy and thus making the prediction more accurate. Concretely, the prediction performance of the hybrid econometric-AI ensemble learning model is illustrated in Fig. 2.

\section{Conclusions}

In this study, a new hybrid econometric-AI ensemble learning methodology is proposed for complex prediction problem. For illustration purpose, the proposed ensemble learning method integrating econometric techniques and AI methods is applied to Chinese export trade prediction problem. Experimental results reveal that the hybrid econometric-AI ensemble learning methodology can significantly improve the prediction performance over other linear and nonlinear models listed in this study, implying that the proposed hybrid econometric-AI ensemble learning methodology can be used as a feasible solution to foreign trade prediction.

\section{Acknowledgements}

This work is supported by the grants from the National Natural Science Foundation of China (NSFC No. 70221001, 70601029), the Chinese Academy of Sciences (CAS No. 3547600), the Academy of Mathematics and Systems Sciences (AMSS No. 3543500) of CAS, and the Strategic Research Grant of City University of Hong Kong (SRG No. 7001677, 7001806).

\section{References}

1. China's Future. Fortune. September 29, 1999

2. Center of China Study: Development, Cooperation, Reciprocal and Mutual Benefit: The Evaluation of International Monetary Fund on Chinese Loan (1981-2002). Research Report (2004)

3. Engle, R.F., Granger, C.W.J.: Co-integration and Error-correction: Representation, Estimation and Testing. Econometrica 55 (1987) 251-276

4. Hendry, D.F., Ericsson, N.R.: An Econometric Analysis of U.K Money Demand in Monetary Trends in the United States and the United Kingdom by Milton Friedman and Anna J. Schwartz. American Economic Review 81 (1991) 8-38

5. Hornik, K., Stinchocombe, M., White, H.: Multilayer Feedforward Networks are Universal Approximators. Neural Networks 2 (1989) 359-366

6. White, H.: Connectionist Nonparametric Regression: Multilayer Feedforward Networks can Learn Arbitrary Mappings. Neural Networks 3 (1990) 535-549

7. Yu, L., Wang, S.Y., Lai, K.K.: A rough-Set-Refined Text Mining Approach for Crude Oil Market Tendency Forecasting. International Journal of Knowledge and Systems Sciences 2(1) (2005) 33-46

8. Sullivan, D.: Document Warehousing and Text Mining: Techniques for Improving Business Operations, Marketing, and Sales. Wiley, New York (2001)

9. Yu, L., Wang, S.Y., Lai, K.K.: A Novel Nonlinear Ensemble Forecasting Model Incorporating GLAR and ANN for Foreign Exchange Rates. Computers \& Operations Research 32 (2005) 2523-2541 